\title{
Optimal Control and Hamiltonian System
}

\author{
Estomih Shedrack Massawe \\ Department of Mathematics, College of Natural Sciences, University of Dar es Salaam, Dar es Salaam, Tanzania
}

Email address:

emassawe2@gmail.com, estomihmassawe@yahoo.com

\section{To cite this article:}

Estomih Shedrack Massawe. Optimal Control and Hamiltonian System. Pure and Applied Mathematics Journal. Vol. 5, No. 3, 2016, pp. 77-81. doi: 10.11648/j.pamj.20160503.13

Received: April 16, 2016; Accepted: April 28, 2016; Published: May 10, 2016

\begin{abstract}
In this paper, an optimal control for Hamiltonian control systems with external variables will be formulated and analysed. Necessary and sufficient conditions which lead to Pantryagin's principle are stated and elaborated. Finally it is shown how the Pontryagin's principle fits very well to the theory of Hamiltonian systems. The case of Potryagin's maximum principle will be considered in detail since it is capable of dealing with both unbounded continuous controls and bounded controls which are possibly discontinuous.
\end{abstract}

Keywords: Optimal Control, Hamiltonian Systems, Conditions for Optimality

\section{Introduction}

It has been essential for many physical systems which are governed by differential equations to be controlled in such a way that a given performance index would be optimized. Large savings in cost have been obtained by a small improvement in performance. The optimal control problem which will be formulated will be the so called Bolza problem [1] with the added condition that the control variables lie in a closed set.

[2] in his paper of Optimal control of stochastic dynamical systems developed existence of stochastic optimal controls for a large class of stochastic differential systems with finite memory is considered. [3] established a feedback control law is developed for dynamical systems described by constrained generalized coordinates. They revealed that for certain complex dynamical systems, it is more desirable to develop the mathematical model using more general coordinates then degrees of freedom which leads to differential-algebraic equations of motion. [4] developed a computational approach to motor control that offers a unifying modelling framework for both dynamic systems and optimal control approaches. In discussions of several behavioural experiments and some theoretical and robotics studies, they demonstrated how the computational ideas allow both the representation of selforganizing processes and the optimization of movement based on reward criteria. [5] proposed a new mathematical formulation for the problem of optimal traffic assignment in dynamic networks with multiple origins and destinations. Several researchers have studied optimal control and dynamical systems. [6] studied Dynamical Systems based optimal control of incompressible fluids. They proposed a cost functional based on a local dynamical systems characterization of vortices. Connections of optimal control and Hamiltonian systems especially the necessary conditions of optimality has not been studied yet. In this paper, it is intended to focus on the link between optimal control and Hamiltonian systems. The case of Potryagin's maximum principle will be considered in detail since it is capable of dealing with both unbounded continuous controls and bounded controls which are possibly discontinuous.

\section{Formulation of Optimal Control Problem}

We consider the state of a control system described by an $n$-vector $\mathbf{x}(t)=\left(x_{1}(t), \ldots, x_{n}(t)\right)$ whose evolution is governed by a system of differential equations

$$
\dot{\mathbf{x}}=g(\mathbf{x}, \mathbf{u})
$$

where $\mathbf{u}$ is a control function from a closed subset of $\mathbb{R}^{n}$ and $X=\mathbb{R}^{n}$.

Given a compact interval $I=\left[t_{0}, t_{1}\right]$, open sets, $X \subset \mathbb{R}^{n}$, 
$V \subset \mathbb{R}^{m}$, a set $U \subset V$ and functions $£, g, L$ and $\phi$ such that

$$
\begin{gathered}
L: X \times V \rightarrow \mathbb{R}, \\
£: X \times V \rightarrow \mathbb{R}, \\
\phi: X \rightarrow \mathbb{R}, \\
g: X \times V \rightarrow \mathbb{R}^{n} .
\end{gathered}
$$

the optimal control problem can be stated as follows:

$$
\operatorname{Minimize} J(\mathbf{x}, \mathbf{u})=\phi\left(x\left(t_{1}\right)\right)+\int_{t_{0}}^{t_{1}} L(\mathbf{x}(t), \mathbf{u}(t)) d t
$$

over all continuous functions $\mathbf{x}$ and measurable functions $\mathbf{u}$ satisfying

$$
\begin{aligned}
& \dot{\mathbf{x}}=g(\mathbf{x}(t), \mathbf{u}(t)), t \in I, \\
& \mathbf{u}(t) \in U, t \in I .
\end{aligned}
$$

$L$ is called the running cost and $\phi$ the terminal cost. [7].

The Pontryagin's principle requires the introduction of the Hamiltonian function $H: X \times \mathbb{R}^{n} \times U \rightarrow U$ given by

$$
H(\mathbf{x}, \mathbf{p}, \mathbf{u})=L(\mathbf{x}, \mathbf{u})+\mathbf{p}^{T} g(\mathbf{x}, \mathbf{u})
$$

In analogy with the corresponding quantity in classical mechanics. $\quad \mathbf{p}_{i}=\left(p_{1}, \ldots, p_{n}\right)=\frac{\partial L}{\partial \dot{q}_{i}} \quad$ is the generalized momenta.

Similar to the formulation of the Hamiltonian systems, the following set of equations hold [8]

$$
\left\{\begin{aligned}
\dot{\mathbf{x}}(t) & =\frac{\partial H}{\partial p}(\mathbf{x}(t), \mathbf{p}(t), \mathbf{u}(t))=g(\mathbf{x}(t), \mathbf{u}(t)) \\
\dot{\mathbf{p}}(t) & =-\frac{\partial H}{\partial \mathbf{x}}(\mathbf{x}(t), \mathbf{p}(t), \mathbf{u}(t)) \\
& =-\frac{\partial L}{\partial \mathbf{x}}(\mathbf{x}(t), \mathbf{u}(t))-\mathbf{p}^{T}(t) \frac{\partial g}{\partial x}(\mathbf{x}(t), \mathbf{u}(t))
\end{aligned}\right.
$$

with boundary conditions

$$
\begin{gathered}
\mathbf{x}\left(t_{0}\right)=\mathbf{x}_{0} \\
\mathbf{p}\left(t_{1}\right)=-\frac{\partial \phi}{\partial \mathbf{x}}\left(\mathbf{x}\left(t_{1}\right)\right)
\end{gathered}
$$

\section{Necessary Conditions for Optimality}

In this section we shall state the necessary conditions for optimality which then lead to Pontryagin's maximum principle.

Theorem

The necessary condition's for $\left(\mathbf{x}_{0}^{*}, \mathbf{u}^{*}(t)\right)$ to be an optimal initial condition and optimal control for the optimal control problem stated above are the existence of a nonzero $k$ dimensional vector $\lambda$ with $\lambda_{1} \leq 0$ and an $n=$ dimensional vector function $\mathbf{P}(t)$ such that for $t \in\left[t_{0}, t_{1}\right]$ : [1]

(i) $\dot{\mathbf{P}}(t)^{\prime}=-\mathbf{P}(t)^{\prime}\left[f_{x}\left(t, \mathbf{x}^{*}(t), \mathbf{u}^{*}(t)\right)\right]$ for $t \in\left[t_{0}, t_{1}\right]$ and $\mathbf{u} \in U$,

(ii) $\mathbf{P}(t)^{\prime}\left[f\left(t, \mathbf{x}^{*}(t), \mathbf{u}(t)\right)-f\left(t, \mathbf{x}^{*}(t), \mathbf{u}(t)\right)\right] \leq 0$,

(iii) $\mathbf{P}\left(t_{1}\right)^{\prime}=\lambda^{\prime} \phi_{x_{i}}(\mathbf{e})$ with $\mathbf{e}=\left(t_{0}, t_{1}, x\left(t_{0}\right), x\left(t_{1}\right)\right)$,

(iv) $\mathbf{P}\left(t_{0}\right)^{\prime}=-\lambda^{\prime} \phi_{x_{0}}(\mathbf{e})$,

(v) $\mathbf{P}\left(t_{1}\right)^{\prime} f\left(t_{1}, \mathbf{x}^{*}\left(t_{1}\right), \mathbf{x}^{*}\left(t_{1}\right), \mathbf{u}^{*}\left(t_{1}\right)\right)=\lambda^{\prime} \phi_{t_{1}}(\mathbf{e})$

(vi) $\mathbf{P}\left(t_{0}\right)^{\prime} f\left(t_{0}, \mathbf{x}^{*}\left(t_{0}\right), \mathbf{x}^{*}\left(t_{0}\right), \mathbf{u}^{*}\left(t_{0}\right)\right)=\lambda^{\prime} \phi_{t_{0}}(\mathbf{e})$

If $f(t, \mathbf{x}, \mathbf{u})$ has a continuous partial derivative $f_{t}(t, \mathbf{x}, \mathbf{u})$ then the condition

(vii) $\mathbf{P}(t)^{\prime} f\left(t, \mathbf{x}^{*}(t), \mathbf{u}^{*}(t)\right)=\lambda^{\prime} \phi_{t_{0}}\left(t_{0}, t_{1}, \mathbf{x}^{*}\left(t_{0}\right), \mathbf{x}^{*}\left(t_{1}\right)\right)$ $+\int_{t_{0}}^{t_{1}} \mathbf{P}(s)^{\prime} f_{t}\left(s, \mathbf{x}^{*}(s), \mathbf{u}^{*}(s)\right) d s$

holds for each $t \in\left[t_{0}, t_{1}\right]$.

Condition (ii) above can be written as

$$
\max \left\{H\left(t, \mathbf{x}^{*}(t), \mathbf{u}\right)\right\}=H\left(t, \mathbf{x}^{*}(t), \mathbf{u}^{*}(t)\right) .
$$

This is called Pontryagin's maximum principle.

The interpretation of this principle is that on the optimal control, $H$ is minimized with respect to the control variables $\mathbf{u}_{j}, j=1, \ldots, m$.

For simplicity we shall treat problem. This is a special case of the problem of optimal control in which the initial time and final time are fixed and there are no conditions on the final state.

We shall restate the Pontryagin's principle so that it fits naturally to our framework of free terminal point problem.

Theorem: (Pontryagin's principle for free terminal point problem) [1]

A necessary condition for optimality of a control $\mathbf{u}$ for the free terminal point problem is that

$$
\mathbf{P}(t)^{\prime}[f(t, \mathbf{x}(t), \mathbf{v})-f(t, \mathbf{x}(t), \mathbf{u}(t))] \leq 0
$$

For each $\mathbf{v} \in U$ and $t \in\left[t_{0}, t_{1}\right]$, where $\mathbf{P}(t)^{\prime}$ is the solution of 


$$
\dot{\mathbf{P}}(t)^{\prime}=-\mathbf{P}(t)^{\prime} f_{x}(t, \mathbf{x}(t), \mathbf{u}(t))
$$

with boundary condition

$$
\mathbf{P}\left(t_{1}\right)^{\prime}=-\phi_{x}\left(\mathbf{x}\left(t_{1}\right)\right) .
$$

The Pontryagin's principle gives only necessary conditions for optimality but these conditions need not be sufficient. Since each optimal control must be external, there must be external controls which are not optimal. However it is natural to ask for conditions which are not optimal. However it is natural to ask for conditions which are both sufficient and necessary for optimality.

Consider a space $v$ of control functions $\mathbf{u}$ defined on $\left[t_{0}, t_{1}\right]$ with values on $\mathbb{R}^{m}$. Let the subset $\kappa \subset \mathcal{V}$ be the set of control functions $\mathbf{u}$ such that $\mathbf{u}(t) \in U$ for each $t \in\left[t_{0}, t_{1}\right]$ and $\left(\mathbf{x}_{0}, \mathbf{u}\right)$ is a feasible pair for the fixed initial state $\mathbf{x}_{0}$. The necessary and sufficient conditions that a control $\mathbf{u}$ be optimal for free terminal point problem is that for each fixed $t \in\left[t_{0}, t_{1}\right]$ we have $P(t)^{\prime} f_{\mathbf{u}}(t, \mathbf{x}(t), \mathbf{u}(t)) \mathbf{v} \leq 0$ for each $\mathbf{v} \in U$ such that $\mathbf{u}(t)+\mathbf{v}(t) \leq \mathbf{0}, \quad K \quad$ is convex and the mapping $J(\mathbf{u})=\phi\left(\mathbf{x}\left(t_{1}\right)\right)$ is a function on $K$. To fit this to the performance index

$$
J(\mathbf{u})=\phi(\mathbf{x}(t))+\int_{t_{0}}^{t_{1}} L(t, \mathbf{x}(t), \mathbf{u}(t)) d t
$$

It is assumed that that $L$ is a real continuously differentiable function and convex in $(\mathbf{x}, \mathbf{u})$ and $\phi$ is continuously differentiable convex function of $\mathbf{x}$. For simplicity we shall consider a linear system.

\section{Theorem}

A necessary and sufficient condition for optimality of a control $\mathbf{u}$ for free terminal point problem with system [1].

$$
\dot{\mathbf{x}}(t)=\mathbf{A}(t) \mathbf{x}(t)+\mathbf{B}(t) \mathbf{u}(t), \mathbf{B} \in \mathbb{R}^{n \times m},
$$

and performance index

$$
J(u)=\phi\left(\mathbf{x}\left(t_{1}\right)\right)+\int_{t_{0}}^{t_{1}} L(t, \mathbf{x}(t), \mathbf{u}(t)) d t
$$

is that for $t \in\left[t_{0}, t_{1}\right]$ :

$$
-L_{\mathbf{u}}(t, \mathbf{x}(t), \mathbf{u}(t)) \mathbf{v}+\tilde{\mathbf{P}}(t)^{\prime} B(t) \mathbf{v} \leq 0
$$

for each $\mathbf{v} \in U$ such that $\mathbf{u}(t)+\mathbf{v} \in U$ where $\tilde{\mathbf{P}}(t)$ is the solution of

$$
\dot{\tilde{\mathbf{P}}}(t)^{\prime}=-\tilde{\mathbf{P}}(t)^{\prime} \mathbf{A}(t)+L_{\mathbf{x}}(t, \mathbf{x}(t), \mathbf{u}(t)),
$$

$$
\tilde{\mathbf{P}}\left(t_{1}\right)^{\prime}=\phi_{\mathbf{x}}\left(t, \mathbf{x}\left(t_{1}\right)\right) .
$$

Moreover if in $L(t, \mathbf{x}, \mathbf{u})$ is strictly convex in $(\mathbf{x}, \mathbf{u})$ for each fixed $t$, the optimal control $\mathbf{u}(t)$ is unique [1].

Proof

Since the corresponding differential equations is a linear system, the set $\kappa$ of controls such that $\mathbf{u}(t) \in U$ and $\left(\mathbf{x}_{0}, \mathbf{u}(t)\right)$ is a feasible pair consists of all piecewise continuous functions such that $\mathbf{u}(t) \in U$. This is a convex set. Let $\mathbf{u}_{0}(t)$ and $\mathbf{u}_{1}(t)$ be controls in $\kappa$ and $\mathbf{x}_{0}(t)$ and $\mathbf{x}_{1}(t)$ the corresponding solutions of the differential equations with $\mathbf{x}\left(t_{0}\right)=\mathbf{x}_{0}$. If $0<\alpha<1$, the convexity of $L(t, \mathbf{x}, \mathbf{u})$ and $\phi(\mathbf{x})$ implies

$$
\begin{aligned}
& \alpha J\left(\mathbf{u}_{0}\right)+(1-\alpha) J\left(\mathbf{u}_{1}\right)=\alpha\left[\int_{t_{0}}^{t_{1}} L\left(t, \mathbf{x}_{0}(t), \mathbf{u}_{0}(t)\right) d t+\phi\left(\mathbf{x}_{0}\left(t_{1}\right)\right)\right] \\
& +(1-\alpha)\left[\int_{t_{0}}^{t_{1}} L\left(t, x_{1}(t), u_{1}(t)\right) d t+\phi\left(x_{1}\left(t_{1}\right)\right)\right] \\
& \quad \geq \int_{t_{0}}^{t_{1}} L\left(t, \alpha \mathbf{x}_{0}(t)+(1-\alpha) \mathbf{x}_{1}(t), \alpha \mathbf{u}_{0}(t)+(1-\alpha) \mathbf{u}_{1}(t)\right) d t \\
& +\phi\left(\alpha \mathbf{x}_{0}(t)+(1-\alpha) \mathbf{x}_{1}\left(t_{1}\right)\right) \\
& =J \alpha \mathbf{u}_{0}+(1-\alpha) \mathbf{u}_{1}
\end{aligned}
$$

since $\alpha \mathbf{x}(t)+(1-\alpha) \mathbf{x}_{1}(t)$ is the solution of the differential equation corresponding to $\alpha \mathbf{u}_{0}(t)+(1-\alpha) \mathbf{u}_{1}(t)$. Therefore $J(\mathbf{u})$ is a convex function on $\kappa$. It can be shown that for each $\mathbf{v}(t)$ satisfying $\mathbf{u}(t)+\mathbf{v}(t) \in U$ for each $t \in\left[t_{0}, t_{1}\right]$ that

$$
\delta J(\mathbf{u}, \mathbf{v})=\int_{t_{0}}^{t_{0}}\left[L_{\mathbf{u}}(t, \mathbf{x}(t), \mathbf{u}(t)) \mathbf{v}(t)-\tilde{\mathbf{P}}(t)^{\prime} \mathbf{B}(t) \mathbf{v}(t)\right] d t \geq 0 .
$$

Hence $J(\mathbf{u})$ has a minimum at $\mathbf{u}=\mathbf{u}(t)[1]$.

If $L(t, \mathbf{x}, \mathbf{u})$ is strictly convex, the above inequality is strict. Thus $J(\mathbf{u})$ is a strictly convex function on $\kappa$ and the minimum is unique.

\section{Connections to Hamiltonian Systems}

To fit the Pontryagin's principle to theory of to the theory of Hamiltonian systems, a control system 


$$
\dot{\mathbf{x}}=g(\mathbf{x}, \mathbf{u}), \mathbf{x} \in X
$$

will be considered with the input space $U$ a manifold without boundaries $H(\mathbf{x}, \mathbf{p}, \mathbf{u})$ and a smooth function in all of its variables. Under these conditions, the Pontryagin's principle implies the first order condition

$$
\frac{\partial H}{\partial \mathbf{u}}=\left(\mathbf{x}, \mathbf{p}, \mathbf{u}^{*}\right)=0 \text { for optimization. }
$$

Consider a simple control system $\sum(X, B, g)$ given by

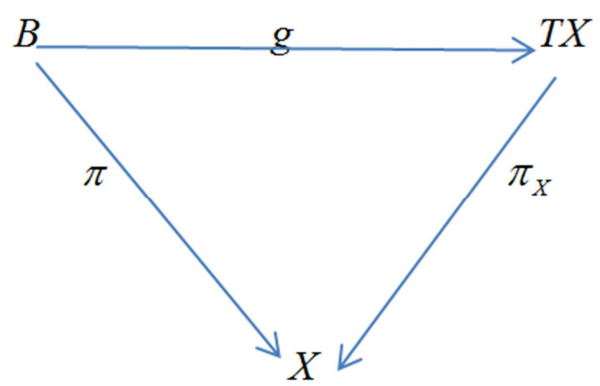

with $B=(\mathbf{x}, \mathbf{u}) . M=T^{*} X$ has a natural symplectic form $\omega=d \theta$ and $T\left(T^{*} X\right)$ has a symplectic form $\dot{\omega}$. The space of external variables $W=T^{*} U=(u, y)$ have a symplectic form $\omega^{e}=d \theta^{e}$. Therefore $T\left(T^{*} X\right) \times T^{*} U$ is a symplectic form $\Omega=\pi_{1}^{*} \omega+\pi_{2}^{*} \omega^{e}$. Let $L: X \times U \rightarrow \mathbb{R}$ be a smooth function and $H: T^{*} X \times U \rightarrow \mathbb{R}$. These functions define the Hamiltonian

$$
H(\mathbf{x}, \mathbf{p}, \mathbf{u})=L(\mathbf{x}, \mathbf{u})+p^{T} g(\mathbf{x}, \mathbf{u})
$$

This is a generating function of the Lagrangian submanifold $N \subset\left(T\left(T^{*} X\right) \times T^{*} U, \Omega\right)$ given by the Hamiltonian equations

$$
\left\{\begin{array}{l}
\dot{x}_{i}=\frac{\partial H}{\partial p_{i}}(\mathbf{x}, \mathbf{p}, \mathbf{u}) \\
\dot{p}_{i}=-\frac{\partial H}{\partial x_{i}}(\mathbf{x}, \mathbf{p}, \mathbf{u}) ; i=1, \ldots, n ; j=1, \ldots, m \\
y_{i}=-\frac{\partial H}{\partial u_{j}}(\mathbf{x}, \mathbf{p}, \mathbf{u})
\end{array}\right.
$$

[8] has shown that Hamiltonian control system is given by

$$
\left\{\begin{aligned}
& \dot{q}_{i}=\frac{\partial H}{\partial p_{i}}(\mathbf{q}, \mathbf{p}, \mathbf{u}) \\
& \dot{p}=-\frac{\partial H}{\partial q_{i}}(\mathbf{q}, \mathbf{p}, \mathbf{u}) ; i=1, \ldots, n ; j=1, \ldots, m \\
& y_{i}=-c_{j} \frac{\partial H}{\partial u_{j}}(\mathbf{q}, \mathbf{p}, \mathbf{u})
\end{aligned}\right.
$$

Comparing equations (11) and (12) it can be concluded that a control system $\sum(X, B, g)$ together with a smooth function $L: X \times U \rightarrow \mathbb{R}$ defines a full Hamiltonian system $\sum\left(T^{*} X, T^{*} U, T^{*} B, f_{H}\right) \quad$ where $\quad f_{H}\left(g_{H}, h_{H}\right) \quad$ with $g_{H}: T^{*} X \times U \rightarrow T\left(T^{*} X\right)$ such that $g_{H}(\mathbf{x}, \mathbf{p}, \mathbf{u})=\left(\mathbf{x}, \mathbf{p}, \frac{\partial H}{\partial \mathbf{p}},-\frac{\partial H}{\partial \mathbf{x}}\right)$ and $h_{H}: T^{*} X \times U \rightarrow T^{*} U$ such that $h_{H}(\mathbf{x}, \mathbf{p}, \mathbf{u})\left(\mathbf{u},-\frac{\partial H}{\partial \mathbf{u}}\right)$. It is assumed that $B$ is a trivial bundle [9].

Let $\operatorname{dim}(X)=n$ and $\operatorname{dim}(U)=m$. Let also $L(\mathbf{x}, \mathbf{u})$, $H(\mathbf{x}, \mathbf{p}, \mathbf{u})$ and the Hamiltonian system $\sum\left(T^{*} X, T^{*} U, T^{*} B, f_{H}\right)$ be as defined above. Then if $\operatorname{rank}\left(\frac{\partial^{2} H}{\partial u_{i} \partial u_{j}}\right)=m, i=1, \ldots, m$ the equation $\frac{\partial H}{\partial u}\left(\mathbf{x}, \mathbf{p}, \mathbf{u}^{*}\right)=0$ has a local Hamiltonian function $H_{o p t}=H\left(\mathbf{x}, \mathbf{p}, \mathbf{u}^{*}(\mathbf{x}, \mathbf{p})\right)$ [10]. We then obtain locally a Hamiltonian vectorfield $X_{H_{o p t}}$ on $T^{*} X$. The projection of the solution curves of $X_{H_{\text {opt }}}$ on $X$ form a set of curves which by Pontyagins principle contains the optimal trajectory $x^{*}($.$) . It is noted that$ $\operatorname{rank}\left(\frac{\partial^{2} H}{\partial u_{i} \partial u_{j}}\right)=m \quad, \quad i=1, \ldots, m$ implies that also $\operatorname{rank}\left(\frac{\partial H}{\partial u}\right)=m$. If we have only rank of the $\operatorname{map}\left(\frac{\partial H}{\partial u}\right)=m$ hen we obtain an immersed Lagrangian submanifold $V$ of $\left(T\left(T^{*} X\right), \dot{\omega}\right)$. This is similar to the implicit Hamiltonian differential equation $P(\mathbf{x}, \mathbf{p}, \dot{\mathbf{x}}, \dot{\mathbf{p}})=0$. If $T\left(T^{*} X\right)$ is projected onto $T^{*} X$ then there may be some points in $V$ where the projection does not have mximal rank and thus the solution of the differential equation will not be defined. If $T^{*} X$ is projected onto $X$, singularities and nonuniqueness of the optimal trajectories my occur [10].

\section{Conclusion}

In this paper, an optimal control for Hamiltonian control systems with external variables has been formulated and analysed. Necessary and sufficient conditions which led to Pantryagin's principle are stated. It was shown how the Pontryagin's principle to the theory of Hamiltonian systems. The case of Potryagin's maximum principle was taken abroad because it is capable of dealing with both unbounded continuous controls and bounded controls which are possibly discontinuous. 


\section{References}

[1] W. H. Fleming and R. W. Rishel, Deterministic and Stochastic Optimal Control, Springer, New York, Inc., 1975.

[2] N. U. Ahmed, Optimal control of stochastic dynamical systems, Information and Control, Volume 22, Issue 1, pp. 1330, 1973.

[3] V. Radisavljevic and H. Baruh, Journal of Dynamic Systems, Measurement, and Control, 121(4), pp. 594-598, 1999.

[4] S. Schaa1, P., Mohajerian1 and A. Ijspeert, Progress in Brain Research, Vol. 165, pp. 425-445, 2007.

[5] S. Lafortune, Introduction to Discrete Event Systems, The International Series on Discrete Event Dynamic Systems, 1993.

[6] M. H. Huller, K. Kunisch, Y. S., S. Volkwein, International Journal for Numerical Methods in Fluids, 00:1-6, 2000.

[7] S. Barnet and R. G. Cameron R. G, Introduction to Mathematical Control Theory, Clarendon Press, 1985.

[8] E. S. Massawe, Hamiltonian Control Systems, International Journal of Theoretical and Mathematical Physics 2016, 6(1): pp. 26-30.

[9] V. der Schaft, System Theoretic Description of Physical System, Doctoral Thesis, Mathematical Centrum, Amsterdam, 1984.

[10] E. S. Massawe, Hamiltonian Control Systems, Unpublished M.Sc Thesis, University of Dublin. 